$36 \mid$ The European strategy for particle physics

\title{
The European strategy for particle physics
}

Particle physics stands on the threshold of a new and exciting era of discovery. The next generation of experiments will explore new domains and probe the deep structure of space-time. They will measure the properties of the elementary constituents of matter and their interactions with unprecedented accuracy, and they will uncover new phenomena such as the Higgs boson or new forms of matter. Longstanding puzzles such as the origin of mass, the matter-antimatter asymmetry of the Universe and the mysterious dark matter and energy that permeate the cosmos will soon benefit from the insights that new measurements will bring. Together, the results will have a profound impact on the way we see our Universe; European particle physics should thoroughly exploit its current exciting and diverse research programme. It should position itself to stand ready to address the challenges that willemerge from exploration of the new frontier, and it should participate fully in an increasingly global adventure.

\section{General issues}

1. European particle physics is founded on strong national institutes, universities and laboratories and the CERN Organization; Europe should maintain and strengthen its central position in particle physics.

2. Increased globalization, concentration and scale of particle physics make a well coordinated strategy in Europe paramount; this strategy will be defined and updated by CERN Council as outlined below.

\section{Scientific activities}

3. The LHC will be the energy frontier machine for the foreseeable future, maintaining European leadership in the field; the highest priority is to fully exploit the physics potential of the LHC, resources for completion of the initial programme have to be secured such that machine and experiments can operate optimally at their design performance. A subsequent major luminosity upgrade (SLHC), motivated by physics results and operation experience, will be enabled by focussed R\&D; to this end, RED for machine and detectors has to be vigorously pursued now and centrally organized towards a luminosity upgrade by around 2015.
4. In order to be in the position to push the energy and luminosity frontier even further it is vital to strengthen the advanced accelerator R\&D programme; a coordinated programme should be intensified, to develop the CLIC technology and high performance magnets for future accelerators, and to play a significant role in the study and development of a high-intensity neutrino facility.

5. It is fundamental to complement the results of the LHC with measurements at a linear collider. In the energy range of 0.5 to $1 \mathrm{TeV}$, the ILC, based on superconducting technology, will provide a unique scientific opportunity at the precision frontier; there should be a strong well-coordinated European activity, including CERN, through the Global Design Effort, for its design and technical preparation towards the construction decision, to be ready for a new assessment by Council around 2010.

6. Studies of the scientific case for future neutrino facilities and the $R \& D$ into associated technologies are required to be in a position to define the optimal neutrino programme based on the information available in around 2012; Council will play an active role in promoting a coordinated European participation in a global neutrino programme.

7. A range of very important non-accelerator experiments take place at the overlap between particle and astroparticle physics exploring otherwise inaccessible phenomena; Council will seek to work with ApPEC to develop a coordinated strategy in these areas of mutual interest. 
8. Flavour physics and precision measurements at the highluminosity frontier at lower energies complement our understanding of particle physics and allow for a more accurate interpretation of the results at the high-energy frontier; these should be led by national or regional collaborations, and the participation of European laboratories and institutes should be promoted.

9. A variety of important research lines are at the interface between particle and nuclear physics requiring dedicated experiments; Council will seek to work with NuPECC in areas of mutual interest, and maintain the capability to perform fixed target experiments at CERN.

10. European theoretical physics has played a crucial role in shaping and consolidating the Standard Model and in formulating possible scenarios for future discoveries. Strong theoretical research and close collaboration with experimentalists are essential to the advancement of particle physics and to take full advantage of experimental progress; the forthcoming LHC results will open new opportunities for theoretical developments, and create new needs for theoretical calculations, which should be widely supported.

\section{Organizational issues}

11. There is a fundamental need for an ongoing process to define and update the European strategy for particle physics; Council, under Article II-2(b) of the CERN Convention, shall assume this responsibility, acting as a council for European particle physics, holding a special session at least once each year for this purpose. Council will define and update the strategy based on proposals and observations from a dedicated scientific body that it shall establish for this purpose.

12. Future major facilities in Europe and elsewhere require collaborations on a global scale; Council, drawing on the European experience in the successful construction and operation of large-scale facilities, will prepare a framework for Europe to engage with the other regions of the world with the goal of optimizing the particle physics output through the best shared use of resources while maintaining European capabilities.

13. Through its programmes, the European Union establishes in a broad sense the European Research Area with European particle physics having its own established structures and organizations; there is a need to strengthen this relationship for communicating issues related to the strategy.
14. Particle physicists in the non-Member States benefit from, and add to, the research programme funded by the CERN Member States; Council will establish how the non-Member States should be involved in defining the strategy.

\section{Complementary issues}

15. Fundamental physics impacts both scientific and philosophical thinking, influencing the way we perceive the universe and our role in it. It is an integral part of particle physics research to share the wonders of our discoveries with the public and the youth in particular. Outreach should be implemented with adequate resources from the start of any major project; Council will establish a network of closely cooperating professional communication officers from each Member state, which would incorporate existing activities, propose, implement and monitor a European particle physics communication and education strategy, and report on a regular basis to Council.

16. Technology developed for nuclear and particle physics research has made and is making a lasting impact on society in areas such as material sciences and biology (e.g. synchrotron radiation facilities), communication and information technology (e.g. the web and grid computing), health (e.g. the PET scanner and hadron therapy facilities); to further promote the impact of the spin-offs of particle physics research, the relevant technology transfer representatives at CERN and in Member states should create a technology transfer forum to analyse the keys to the success in technology transfer projects in general, make proposals for improving its effectiveness, promoting knowledge transfer through mobility of scientists and engineers between industry and research.

17. The technical advances necessary for particle physics both benefit from, and stimulate, the technological competences available in European industry; Council will consolidate and reinforce this connection, by ensuring that future engagement with industry takes account of current best practices, and continuously profits from the accumulated experience.

Unanimously approved by the CERN Council at the special Session held in Lisbon on 14 July 2006 